\title{
INTEGRATION OF ARTIFICIAL INTELLIGENCE WITH CLOUD SERVICES
}

\author{
Aleksandar Petrović*, \\ Miodrag Živković
}

\section{Singidunum University,}

Belgrade, Serbia

\section{Correspondence:}

Aleksandar Petrović

e-mail:

aleksandar.petrovic.17@singimail.rs

\begin{abstract}
:
Recently, we have entered a new era, which is known as the cloud era. Interest in cloud computing continues to increase, and many companies have already moved their IT in the cloud. On the other hand, advances in artificial intelligence opened new possibilities which can be used to improve current cloud technologies. This paper explores those possibilities and what is already achieved with the integration of artificial intelligence and cloud-based storing solutions. A brief explanation of both technologies is given, followed by a more widened approach to the benefits they achieve together. Analysis of social as well as of financial benefits is presented. We approached these possibilities and indicated the ways in which artificial intelligence can be fused with cloud storage. Solutions to the obstacles to these technologies are suggested through their examination. Further research and development options are submitted while instructing caution for the damage artificial intelligence and cloud could do together.
\end{abstract}

Keywords:

artificial intelligence, cloud, innovation, productivity.

\section{INTRODUCTION}

Productivity challenges and economic data storage were observed as a large problem for the corporations until cloud storage was introduced. Cloud was not immediately considered as a serious solution to this problem. However, the cloud has striven and became one of the most important and fundamental technologies on which other technologies rely on. Besides backup, high level of organization was essential for big databases. Artificial intelligence has first been applied for organizational matters of the cloud and then became essential to it. Currently, the most advanced cloud platforms are those that are fused with the power that AI (artificial intelligence) offers. Artificial intelligence is also important for big data analytics and it strived with cloud and AI advancements. Big data is important because humans exchange uncountable units of data in real time, not to mention the amount of data that was generated historically. This information is invaluable if approached correctly. The space for improvement of society's standards is wide but the focus is on getting more for less. Cloud and AI give society a better value for money compared to the 
traditional technologies in the past. Better organization, achieving access to information that was not accessible before, and keeping information safer than before is the goal of these technologies that are the pillars of today's information technology world.

\section{BACKGROUND}

\section{Cloud}

Elementary use of cloud services is relying on the scalability of data storage, as any required capacity can be achieved without planning and at any point in time. This eliminates the number of servers and their capacity prediction for the reason that the service providers always offer possibilities to increase or even decrease the capacity of storage and to manage other services that the client is paying for. Cloud platforms have a much higher potential than just storing data due to distributed computing power. The more important part of the cloud's scalability is the ability to distribute more hardware resources to a certain user when it is required. These situations occur when it is needed to store more data or more processing power is required from a service that is provided. Users only pay for the resources that they have been using, those resources being more storage or power. Migration of the services to the cloud greatly reduces the costs of any organization. Servers and additional required equipment are costly, especially when it comes to large scale operations. The implementation of the cloud removes the need for server management and reduces maintenance costs. Productivity and efficiency benefits are paramount when considering all the advantages that cloud offers. Vast benefits include always accessible data which provide the flexibility to widen the availability of support services and to remove limits from using only the on-premises equipment. Always having access to provided services requires replicating and distributing data to more than one cloud server and that redundancy is one more service that is achieved through the cloud, backup. Containerization of applications is another focal cloud service [1]. Instead of virtualizing complete operating systems, containers are made from the application layer. This allows for easy application distribution through the cloud and improved storage efficiency. Containerization provides more flexibility and utilizes resources better than traditional virtualization. As machine learning was getting more sophisticated it started offering opportunities for cloud servers to exploit including artificial intelligence. Cloud providers, working with massive amounts of data, acknowledged the opportunity to develop self-improving algorithms. Amazon provides advanced analytics service as one of the basic services of its extensive ever-developing AI arsenal [2].

\section{Artificial intelligence}

Artificial intelligence technologies are becoming more sophisticated and as they are getting better they are also posing a bigger threat to humans if they become mishandled. AI technology is being slowly introduced to the general masses in the forms of deep learning enhanced devices as well as optimizing smartphone hardware for AI-based software. Examples of this kind are Amazon's DeepLens, which is able to recognize human emotions from analyzing photographs, and an application Homecourt [3], that watches while the subject plays and it makes personalized suggestions. Smartwatches use AI in combination with body sensors to detect possible unintended falls of the subject, high blood pressure, and even possible diabetes. Commercial usage is still in its early stage but the fact that they are making their place in the market is encouraging. Systems with integrated or hardware enabled AI are able to learn from their mistakes and adapt to different problems that are presented to them. The learning process concerning artificial intelligence is called machine learning, or on a higher level, the evolution of machine learning, the deep learning. Machine learning is primitive compared to deep learning and consists of algorithms that analyze data, extract knowledge from it, and then present a solution to the given problem [4]. This process grants a trained machine capable of completing the task that would otherwise require hours of coding. Some of the learning methods are decision tree learning, inductive logic programming, clustering, and reinforcement learning. Deep learning is different in a way that it layers algorithms with the goal of creating an artificial neural network. Neural networks are attempting to imitate the human neural system with an emphasis on the way human neurons connect with each other. In comparison to natural neural networks, artificial ones are made of layers, connections, and directions of data propagation. Artificial neurons carry a factor of correctness concerning the data they are analyzing. When every neuron is put into the equation total outcome is calculated by the feedback of each neuron. None of these methods achieved the goal of general AI (artificial intelligence) [5], qualified for completing any type of task. Striving for automatization of as many jobs as possible, especially 
jobs that are hazardous, led to implementing AI to the robots. AIs are superior to humans when it comes to carrying out defined tasks, but they are not immaculate. Their performance depends on the level of training they have been through and how long did it last. The learning process is to tell them where they went wrong and to let them figure out on their own how to overcome it. The danger of having everything automatized is frightening the society but is also what everybody wants. Many professions will go extinct but the machines working for us should not be considered immoral. This field is not fully explored nor defined. Standards need to be applied but that is only possible when complete understanding of artificial intelligence is achieved.

\section{THE SYMBIOSIS OF AI AND CLOUD}

Artificial intelligence needs to learn and to be trained, and there are two methods, supervised and unsupervised learning [6]. With supervised learning, AIs are presented with training datasets, that are boundaries of a correct outcome of the operation. The structure of a dataset consists of basic facts about the objects in the problem. Should a returned value appear incorrect, a supervisor will intervene and direct the AI to the correct solution of the problem. The difference concerning unsupervised learning is that training datasets and outcomes are not defined. Their purpose is to solve complex problems with binary logic while only using the submitted data. Returned values are not systemized exclusively as correct and incorrect answers. Every answer is calculated to a degree of probability. Semi-supervised learning is in most cases an acceptable solution to the needs of an average client. This occurs due to incomplete or inaccurate data on the matter. The combination enables usage of reference data when it is available and applies calculated probability when such data is nonexistent. Various roles [7] can be assigned to an AI in retail, supply chains, news, financial services, healthcare, etc., but what is undoubtedly needed are massive amounts of data [8]. Adaptation of content in advertisements to the needs of every user, optimization of routes, prediction of changes in demand, are data demanding processes, but the biggest potential and the highest requirements are held by the healthcare industry. AI integration with the smart scanners could provide automatized visual diagnostics, reduce the maintenance expenses, reduce the human error, enable robotized surgical assistance, and improve the data keeping.

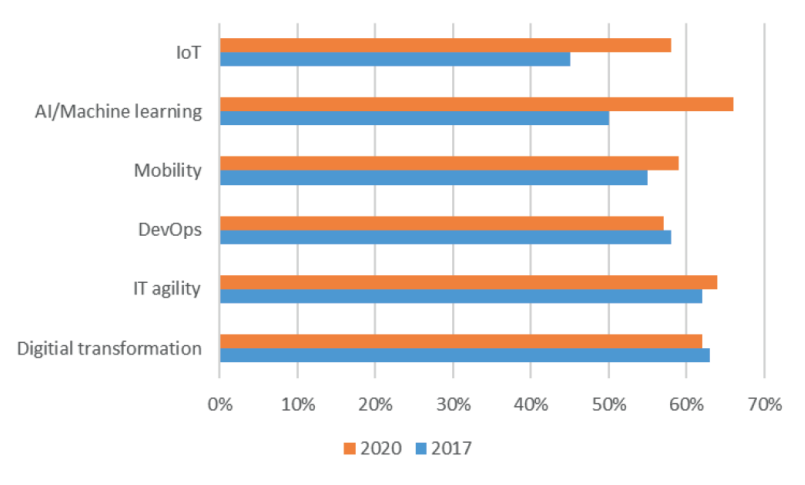

Fig. 1. Factors driving public cloud engagement in the United States in 2017 and 2020

Possibilities of connecting an AI to a cloud have various benefits to both sides. Cloud servers hold a lot of data and that is an invaluable resource to any AI, but if more AIs are connected, they can learn from each other's mistakes. When one particular AI learns a process, it can easily transfer the knowledge to other AIs, which drastically increases the potential of such symbiosis. In the past, AI's growth was hindered due to limited datasets, it was unable to analyze all the real-time data. Big data is the fuel that powers AI. Advancement of big data analytics removed these obstacles. Tools have been created that enable rapid analysis and technology is now agile enough to access colossal datasets. Due to the cloud's scalability, AI services provided from the cloud are as well scalable. When an enterprise wants to expand AI concerning projects the cloud permits adding computing power or requesting more hours of the full capacity of servers from the providers [9]. Renting AIs is a great way of getting access to advanced computing power while minimizing the costs by not paying for all the infrastructure and by accomplishing highest efficiency because of paying only for the time or percentage of the power used. Cloud AI is easier to use because AIs need to be trained, as said before, and that development process is very expensive. It is a goal to implement an $\mathrm{AI}$ in all cases where it can increase efficiency. AIs are great for completing various tasks, but they are usually specialized for one task only. The most popular use is with tremendously large databases. Huge databases need sorting, which calls for machine learning. Analytics tools offer classification and superior organization. Prior to the implementation of artificial intelligence with data analysis methods analysts spent more time on preparing the data than on its analysis. The innovation AI brought was with the automatic ingestion of data, classification, and organization of all data sources. 


\section{EXAMPLES}

In the next chapter, we reviewed the most popular cloud AI solution providers, the variety of the services they provide, their influence on the current market, and the leverage each one possesses. The dominating companies in AI development are the first comers that invested largely into cloud architecture because their main business, or at least one of the top five most important businesses, was working with enormous amounts of data. These companies accumulated unthinkable amounts of information as results of gathering it so it could be provided on demand, users of its services generating massive amounts of it, and even for staying the longest in the business. Based on their main fields the companies specialized their AI in different ways. The one adoption of AI that most of them are developing is text-to-speech and vice versa, a comparison of these services will be presented into detail. The slightly different competitor is certainly Google for developing its specialized processing unit for AI that brings the hardware advantage over the other companies. More word on this project in the following paragraphs. Besides the scientific contribution from Google, Amazon is the leader in consumer AI, but the way the market is developing is sometimes proving better in a couple of ways to even develop private cloud networks with AI, but more word on this in Chapter IV, and the most dominant in the field of independent developers is Microsoft for its widespread systems over the years that it has been active. There is a fair market of companies that are offering cloud computing services but there are also those that are not embracing the trend. The most worrisome behavior comes from Apple. The company has been renting Microsoft's cloud infrastructure for a long period of time before transitioning onto Google's infrastructure. This profoundly hurts Apple's finances and there have been rumors of Apple Pie project, of which very little is known, but considering Apple's behavior of ignoring the tech trends and later releasing a much better version of them, this does not extinguish all hope. It is certainly disappointing to watch Apple sit this one out on the bench because of the great success their personal assistant, Siri, achieved. The other companies worth mentioning besides the giants like Google and IBM are Oracle AI, Salesforce and Baidu.

\section{Amazon}

The most consumer and profit-oriented are certainly the AWS (Amazon Web Services). This is not a surprise, taking into account that the company operates in the trading market and that its main principle is supply and demand. However, a common user of the large online marketplace should not underestimate the company and think it has not evolved into a tech giant. The substantial revenue generated needed to be reinvested and the natural way was to explore advanced online advertising and algorithms to suggest similar and products of interest to the certain user. Amazon's Kindle, Prime, and $\mathrm{Mu}-$ sic easily revealed that the company is expanding from trading. The company later introduced its cloud services that became a huge success. Gaining momentum, the company largely widened the scope of services that were covered with AWS. One of the services that the users most familiarize with is Amazon Personalize. The service is based as an advanced tool for product placement based on an over the years perfected machine learning algorithm. The main benefit is that it allows exploitation of machine learning in software solutions with extensive prior knowledge of it. The user pays only for the time that has been used to process the data that is forwarded to it. Besides software services, Amazon has to offer AI optimized hardware such as DeepLens, a deep learning camera that can detect an extensive range of human emotions from the footage it captures. DeepLens comes with an online library of pre-trained deep learning modules, and some of them are trained to recognize a cat or a dog, a face, an activity, and the name of the object. This AWS is compatible with SageMaker, Amazon's machine learning developer studio, which provides great compatibility. The most successful Amazon service and the product is Alexa. Exploiting Amazon Web Services like Polly and Rekognition, Alexa became a powerful personal assistant that is more popular on the market than the competing companies' personal assistants [10].

\section{Microsoft}

The second biggest cloud AI provider is Microsoft. The leverage Microsoft has when it comes to the machine and deep learning is the immense number of devices that it has in its ecosystem. Set aside the diversity Microsoft is one of the companies with the largest numbers of devices that run its operating system. A large number of Windows-based hardware allowed a smooth introduction of Azure, Microsoft's cloud computing platform in 2010. Microsoft developed its own cloud computing service that supports many different programming languages with an accent on development. Azure possesses environments for building, testing, 
managing, and deploying software solutions. The most important available tools included are the Visual Studio with a tool for AI, Azure Machine Learning Studio, and AI Toolkit for Azure IoT Edge. Azure also provides an excellent text-to-speech service.

\section{Google}

Google's role in developing AI through the cloud is not as high profile as Azure or AWS, but the contribution of this data giant is far from mediocre. Like other competitors, Google provides visual recognition, speech-to-text, text-to-speech, translations, and a machine learning engine. Google's edge is undoubtedly the biggest data flow and the amount of data collected. An interesting twist to Google's video deep learning is the ability to search for text and even images in the video. Besides, the obvious advantage of acquiring the most data Google's biggest leverage is its self-developed processing unit specialized for its machine learning engine. It is called TPU (Tensor Processing unit) and it provides from fifteen to thirty times faster performance than any CPU or GPU [11].

\section{$I B M$}

The tech giant has not been in the spotlight for a long time so it is unexpected for it to have the most diverse cloud computing services with around a hundred and seventy services. The substantial number of services the company owns to the grouping of its BlueMix cloud service, SoftLayer data centers, and Watson AI group. Watson provides highly sophisticated analytics services, speech-to-text conversion, various translations scenarios, and visual recognition.

\section{Charts}

The charts we presented show how many companies use certain providers' infrastructure and the usage of the most popular personal assistants. It is important to remember that the users' choices heavily influence the future of technological development. It is interesting that Microsoft is right behind Amazon in cloud computing and that Google is lacking so much in comparison to the prior two. It was expected of Apple's personal assistant to dominate the market considering Apple's smartphone dominance, but Google proved the power of the Android platform and fortified its second-best position.

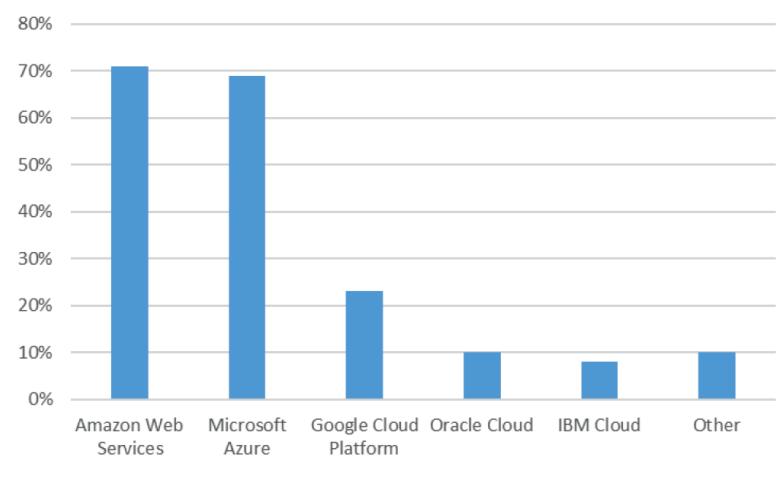

Fig. 2. Cloud Infrastructure as a Service (IaaS) vendor currently in use in organizations worldwide as of 2018

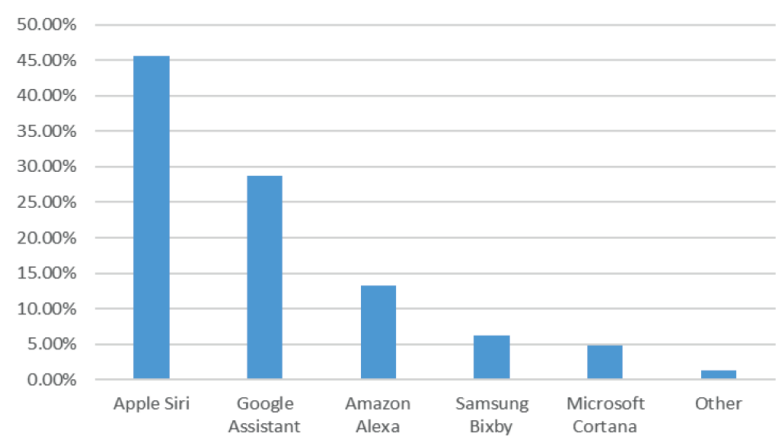

Fig. 3. Market share of voice assistants in the U.S. (May 2018)

\section{OBSTACLES FOR FUTURE IMPROVEMENT}

\section{Technical}

The merging of AI and cloud resulted in a significant increase in efficiency and innovation potential [12]. While operations expand, larger data workloads are worked with, and possibilities are perceived to reduce the expenses. Intelligent data storage layer on the cloud offers high efficiency and scalability that are mandatory solutions to this problem. Cloud has a vast set of tools that are striving to securely analyze data economically. Distinct ways [13] of data processing offer different results in the prioritization of real-time data or the focus on being as accurate as possible. It is difficult for the most advanced systems to keep up with the amounts of data that is generated at the moment and it is even more time consuming to analyze all the data on a certain subject. Management of exponential data growth in a scalable, efficient way is the goal and that is why datasets 
are put together. The purpose of a dataset is to contain analyzed data only on the matter that is of interest at that moment. AI can have a massive impact on the preparation of datasets and simplifying the job for humans. More powerful infrastructure is required for fined tuned results and that is why computing power is a serious obstacle for artificial intelligence and cloud as well. Both branches need very sophisticated infrastructure to provide the services they offer, especially if such systems are developed privately. Establishing private cloud networks that integrate AI, besides the infrastructure costs yields costs as big in human resources. Regular maintenance and supervision crews are expensive due to the essential always-online support and the expenses multiply when a group of experts is assigned to train an AI. In spite of the obvious disadvantages, developing a private cloud computing network can be more cost-effective if there is a need for cloud computing service to run frequently. Creating a general AI is attempted and closest the general effort has gotten is the multitasking [14] methods which are divided by the way they connect tasks to each other. Tasks can be divided after connecting all the data on them or the data on each task can be divided and later connected when the tasks are completed separately. AI's biggest disadvantage currently is the inability to deal with different occupations. General artificial intelligence is what is thought about when it is talked about AIs. Currently, the AIs that are available are very sophisticated algorithms with a possibility of expansion under certain conditions, which is the main reason why the actual perfect assistant cannot yet be implemented.

\section{Legal}

Artificial intelligence has been encountering problems with the various governments. The law is getting twisted when it comes to criminal liability and jurisdiction that it would have [15]. Some have redefined their laws according to the AI, but those regulations are only a work in progress. Legal workers have been battling to achieve controlled access to permissions that the AI technologies need without violating human rights [16]. Examples of practical usage include image processing, geotagging [17], three-dimensional environment processing, speech analysis, and data mining. The examples listed above need access to cameras, microphones and a lot of historical data. Algorithms are used to analyze images to extract information as well as in real time with the autonomous vehicles that require radar and laser data to understand three-dimensional geometries. Text analysis has even more application because of the massive amounts of data being generated daily. It is used to obtain information, apply a classification or to pull out particular pieces of data. This sort of analysis is also considered as data mining. Large quantities of data are needed to reap the full benefits of the analytics tools. Individual's security is questioned when a lot of data is analyzed, especially because confidential data is needed for some analysis. The sensitive analyzed data is valuable to hackers and if carelessly stored and used can be a threat. In certain cases storing that kind of data on the cloud is unpermitted and there are also limitations to where the data is stored geographically. International companies that handle personal information that is necessary for its services to function properly are having problems with countries that have strict privacy laws. Most countries are not allowing storage of personal data of its citizens on cloud servers that are geographically in different countries. When simplified to its lowest level, keeping sensitive information on hardware that is out of legal jurisdiction from its country of origins is violating a lot of constitutional rights. When presented in this way there is no doubt that it could be argued that is unacceptable to handle sensitive information like that, but when put into perspective, an individual shares a considerable amount of personal information on the web and the direction that digital privacy should be taken into is education, awareness, and simplifying the means to protect oneself.

\section{CONCLUSION}

Innovations that the combination of cloud and artificial intelligence brought can be of great importance to society. Raising the standard for human lives is a philanthropic deed that we should all commit to. In the present-day devices with sophisticated software are growing and multiplying exponentially, tons of data are injected into the systems. The corporate world has a wide spectrum of opportunities that would not be presented to them if it were not for the analysis of massive amounts of information. For some time, it has been a common practice for operations to store important and even all of the data in the cloud. Artificial intelligence gives wings to the organization and classification methods of that vary storage. The inventors should continue perfecting the solutions that are currently used and with great effort, a general AI could see the light of day. AI must not be underestimated and neglected because it is at this moment a double-edged sword. That is why the 
legal regulations should be prepared even before. It is a high risk but it cannot be said with certainty that it will bring a high reward.

\section{REFERENCES}

[1] Q. Zhang, L. Liu, C. Pu, Q. Dou, L. Wu, W. Zhou, "A Comparative Study of Containers and Virtual Machines in Big Data Environment", article

[2] S. Mathew "Overview of Amazon Web Services", whitepaper

[3] K. Leswing, Business Insider, "Ex-Apple engineers created an app to track basketball shots using AI, and it's already getting attention from Mark Cuban and the NBA", blog

[4] J. Le "The 10 Deep Learning Methods AI Practitioners Need to Apply", blog

[5] J. McCarthy, Stanford University, "Generality in Artificial Intelligence", paper

[6] P. Mell, T. Grance, NIST, "The NIST Definition of Cloud Computing", paper

[7] H. Motahari-Nezhad, B. Stephenson, S. Singhal, HP Laboratories, "Outsourcing Business to Cloud Computing Services: Opportunities and Challenges", article
[8] S. Pearson, HP Laboratories, "Taking Account of Privacy when Designing Cloud Computing Services", article

[9] C. Harvey, "AI in the Cloud Boosts Cloud Performance", online article

[10] Google, "https://trends.google.com/trends/explore? $\mathrm{q}=$ Alexa,Siri,Cortana", statistic

[11] N. P. Jouppi, C. Young, N. Patil, D. Patterson, Gaurav Agrawal, and others, IEEE, "In-Datacenter Performance Analysis of a Tensor Processing Unit", paper

[12] AI Impacts, "Trends in the cost of computing", scientific analysis

[13] S. Riesen, Technologist, "The challenges of artificial intelligence", magazine

[14] S. Ruder, "An Overview of Multi-Task Learning in Deep Neural Networks", blog

[15] B. Buchanant, T. Headrickt, Stanford Law Review, Volume 23, No. 1, November 1970, Board of Trustees of the Leland Stanford Junior University, "Some Speculation About Artificial Intelligence and Legal Reasoning”, article

[16] Enterprise Innovation, "2018: The rise of AI, hybrid cloud and digital human rights issues", magazine

[17] S. Agarwal, J. Dunagan, N. Jain, S. Saroiu, A. Wolman, University of Toronto, "Volley: Automated Data Placement for Geo-Distributed Cloud Services", article 